\title{
Una primera aproximación a la comprensión que tienen estudiantes universitarios en Chile de la Teoría de la Evolución*
}

\author{
An initial approach to Chilean undergraduate students' understanding \\ of the Evolution Theory \\ Uma primeira aproximação da compreensão que têm estudantes universitários no \\ Chile a respeito da Teoria da Evolução
}

\section{Hernán L. Cofré, ${ }^{a, b}$ Claudia A. Vergara, ${ }^{a, c}$ David P. Santibáñez, ${ }^{d}$ Juan P. Jiménez ${ }^{a}$}

${ }^{\text {a}}$ Illinois Institute of Technology, Mathematics and Science Education Department, Chicago, IL, USA. Correo electrónico: hernan.cofre@ucv.cl

bPontificia Universidad Católica de Valparaíso, Instituto de Biología, Av. Universidad 330,

Curauma, Valparaíso, Chile.

'Universidad Alberto Hurtado, Facultad de Filosofía y Humanidades, Santiago, Chile.

¿Universidad Católica Silva Henríquez, Facultad de Educación, General Jofré 462, Santiago, Chile.

\begin{abstract}
RESUMEN
Dada la importancia que tiene la Teoría de la Evolución (TE) como conocimiento científico, el objetivo del presente estudio fue caracterizar la compresión sobre la TE de un grupo de 248 estudiantes universitarios. Los datos fueron recolectados en base a un instrumento de 18 preguntas (3 demográficas, 14 de escala likert y una abierta), el cual se enfocó en tres tópicos: la comprensión sobre los conceptos de Teoría y Ley (CTL), la comprensión de la TE (CTE) y la aceptación de la TE (ATE). El análisis de los datos reveló que el $74 \%$ de los participantes acepta la TE como un conocimiento científico establecido, sin embargo, también presentan errores conceptuales sobre la TE y CTL. Las implicancias de este estudio para la enseñanza de la TE en la educación secundaria y para la formación de profesores de ciencia también son discutidas.
\end{abstract}

Palabras clave: evolución, comprensión de la evolución, aceptación de la evolución, errores conceptuales, naturaleza de la ciencia.

\begin{abstract}
Given the importance of the Theory of Evolution (TE) as scientific knowledge, the aim of the present study was to characterize the understanding about TE of 248 undergraduate students. Data was collected based on an 18 question instrument (3 demographic, 14 Likert scale and one open-ended), that targeted three main topics: Understanding of Theory and Law (UTL), Understanding of TE (UTE), and Acceptance of TE (ATE). The analysis of data revealed that $74 \%$ of the participants accept TE as established scientific knowledge; however, they also had misconceptions about ET and TL. The implications for teaching TE in high school and in the education of science teachers are also discussed in this study.
\end{abstract}

Key words: evolution, understanding of evolution, acceptance of evolution, misconceptions, nature of science.

\section{RESUMO}

Dada a importância da Teoria da Evolução (TE) enquanto conhecimento científico, objetiva-se caracterizar a compreensão de 248 estudantes de licenciatura sobre a TE. Os dados foram obtidos por meio de um instrumento de 18 perguntas

* La etapa de análisis y escritura de este trabajo se desarrolló en el marco de dos Becas Chile de Post doctorado al primer autor y la segunda autora. 
(3 demográficas, 14 de escala de Likert e 1 de resposta aberta), a qual focou três tópicos: Compreensão dos conceitos de Teoria e Lei (CTL), Compreensão da TE (CTE), e Aceitação da TE (ATE). A análise dos dados revelou que 74\% dos participantes aceitam a TE como um conhecimento científico estabelecido, mas também apresentam concepções equivocadas sobre a ET e CTL. As implicações do estudo para o ensino da TE na Educação Secundária e na formação de professores de Ciências também são discutidas.

Palavras chave: evolução, compreensão da evolução, aceitação da evolução, equívocos conceituais, natureza da ciência.

Nothing in Biology makes sense except in the light of evolution Dobzhansky, T. (1973), The American Biology Teacher

\section{INTRODUCCIÓN}

Pese a ser considerado uno de los conocimientos científicos indispensables para lograr el objetivo de la alfabetización científica en el mundo (Harlen, 2010), numerosos estudios realizados en las últimas décadas han revelado un nivel preocupante de errores conceptuales en la comprensión de los principios básicos de la Teoría de la Evolución en estudiantes, profesores y público en general (e.g. Miller et al., 2006; Nehm y Schonfeld, 2007; Kampourakis y Zogza, 2007; McFadden et al., 2007; Gregory y Ellis, 2009; Gregory, 2009; Gutiérrez, 2009; Kim y Nehm, 2010). Esta falta de comprensión de la Teoría de la Evolución (TE en lo que sigue), muchas veces va acompañada de un sentimiento de incredulidad y rechazo de ella, el cual tiene su mayor expresión en ciertas regiones de Estados Unidos y en algunos países de Europa oriental (Miller et al., 2006). El evaluar esta falta de aceptación y comprensión de la TE, así como el detectar cuáles son los principales problemas que enfrentan los estudiantes para comprender este contenido científico y como mejorar su enseñanza, son algunos de los temas más relevantes hoy en día en el área de la enseñanza de la biología en el mundo (e.g. McFadden et al., 2007; Nehm y Reilly, 2007; Lombrozo et al., 2008; More, 2008; Gregory, 2009; Kim y Nehm, 2010; Smith, 2010a).

En Chile, el currículo nacional de ciencias incluye el tema de la TE desde el octavo año básico (MINEDUC, 1998, 2009). No obstante, desde el segundo ciclo (5º́sico) comienza la revisión de conceptos relacionados como: adaptación, población, especie, biodiversidad, entre otros, para terminar en octavo básico, con la revisión de los orígenes de la vida y la historia evolutiva de los diferentes grupos de organismos a través del registro fósil (MINEDUC, 2009). Sin embargo, la mayor cobertura y profundidad se alcanza en la enseñanza media, donde se revisan temas de variabilidad genética, herencia, selección natural, evolución humana, entre otros (MINEDUC, 2009). Esta mayor cobertura se alcanzó luego de varias décadas en que el estudio de la evolución estuvo muy disminuido dentro de los contenidos (Medel, 2008; Camus, 2009; Tamayo y González, 2010). Por otra parte, si bien existen algunos aportes sobre el tratamiento de los contenidos de la TE, ya sea en el currículo nacional (Medel, 2008; Camus, 2009; Tamayo y González, 2010), como en los libros de texto (Tamayo y González, 2010), y también una genuina preocupación por mejorar el entendimiento de la evolución en Chile por parte de los científicos dedicados a esta disciplina (e.g. Spotorno, 1991; López y Spotorno, 2008; Camus, 2009; Gallardo, 2011), hasta la fecha no existe ninguna investigación que dé cuenta de cuál es el grado de aceptación que presentan nuestros estudiantes y el público en general, ni de cuál es el entendimiento que tenemos de esta gran idea científica en 
los diferentes niveles educativos. Con este trabajo se comienza a llenar este vacío de información en un área esencial de la enseñanza de la Biología.

\section{MARCO TEÓRICO Y REFERENCIAL}

La TE es el principio unificador de la Biología (Dobzhansky, 1973; Mayr, 2001). Sin embargo, aún en este siglo existe un gran desconocimiento sobre sus mecanismos y principales postulados e incluso en cierta parte de la población persiste un sentimiento de rechazo e incredulidad. Por ejemplo, Miller et al. (2006) mostraron que en Estados Unidos y algunos países de Europa oriental como Letonia, Chipre y Turquía el grado de aceptación de la TE por la gente adulta era menor al 50\%. Esta falta de aceptación, al menos en los Estados Unidos, está asociada al fundamentalismo religioso y la politización que se ha hecho del tema (Miller et al.,, 2006).

Desafortunadamente, este panorama no es mucho mejor cuando se estudian las concepciones y conocimientos sobre evolución que poseen los profesores de biología en preparación o en ejercicio (Kim y Nehm, 2010), o los estudiantes universitarios de carreras asociadas a la biología (Alter y Nelson, 2002; Nehm y Reilly, 2007; Mc Fadden et al., 2007; Gregory y Ellis, 2009; Gregory, 2009; Gutiérrez, 2009). Por ejemplo, Kim y Nehm (2010) en una revisión sobre el tema, mostraron que la aceptación de la TE en los profesores de biología llega a niveles bajos en países como Estados Unidos y Turquía, donde no sobrepasa el 60\%. Por otra parte, países de Europa muestran menores niveles de rechazo en los profesores de biología, pero estos aun se mantienen cercanos al $20 \%$ en Alemania, Austria, Suiza o Inglaterra.

Por otro lado, numerosos trabajos han mostrado que el grado de conocimiento y comprensión de la TE que tienen tanto los profesores como estudiantes universitarios, son muy bajos, encontrándose una relación positiva entre la comprensión de los contenidos y la aceptación de la TE como una explicación científica (Mc Fadden et al., 2007; Gregory y Ellis, 2009; Gregory, 2009; Gutiérrez, 2009; Kim y Nehm, 2010). En cuanto al conocimiento y la comprensión que manejan estudiantes universitarios, si bien se ha mostrado que son el grupo más informado dentro de la sociedad, mejor que estudiantes secundarios y público en general (Mc Fadden et al., 2007; Gregory y Ellis, 2009), también se ha mostrado que aquellos que no comprenden bien los mecanismos evolutivos, mantienen errores conceptuales muy similares a los de estudiantes más jóvenes (Alter y Nelson, 2002; Gregory y Ellis, 2009; Gregory, 2009; Pazza et al., 2010), incluso después de asistir a cursos en la universidad (Nehm y Reilly, 2007).

En cuanto a los errores conceptuales asociados a la enseñanza de la TE, se ha mostrado que los estudiantes con frecuencia atribuyen el cambio biológico a una respuesta voluntaria del organismo a una nueva necesidad impuesta por el medio ambiente (Rudolph y Stewart, 1998; Kampourakis y Zogza, 2007; Gregory, 2009; Gutiérrez, 2009). Esta explicación intuitiva o ingenua de los estudiantes, se basa en un pensamiento Teleológico (Mayr, 2001; Kampourakis y Zogza, 2007) y ha sido clasificada erróneamente por muchos investigadores como una preconcepción "Lamarckista" (Rudolph y Stewart, 1998, véase además Kampourakis y Zogza, 2007 para una revisión). Esta clasificación incorrecta puede dificultar la generación de estrategias de enseñanza que favorezcan la comprensión de los mecanismos correctos asociados a la TE (Kampourakis y Zogza, 2007). Por esta razón, 
en este trabajo se seguirá la proposición de Kampourakis y Zogza (2007), en términos de distinguir entre una visión ingenua de: evolution through need via purpuseful change, de explicaciones genuinamente Lamarckistas: evolution through the habit of use y Darwinistas: evolution through differential reproduction and survival base don population variability (véase además Gregory, 2009). Por otra parte, está claro que, pese a estos esfuerzos de caracterización de errores conceptuales, las visiones y explicaciones alternativas de los alumnos son mucho más complejas, las cuales pueden incluir combinación de las ideas intuitivas, Lamarckistas y Darwinistas (Gutiérrez, 2009).

A este complejo escenario de comprensión de la TE, se suma el hecho de que existen evidencias de que una visión tradicional y poco elaborada de la naturaleza de la ciencia puede influir de forma negativa en la comprensión y, especialmente, en la aceptación de los postulados de Darwin (e.g. Dagher y BouJaoude, 2005; Hokayem y BouJaoude, 2008; Lombrozo et al., 2008; Gregory, 2009; Kim y Nehm, 2010; Smith, 2010ab). Específicamente, el pensar que el nuevo conocimiento sólo se genera mediante experimentos, que las teorías son conocimientos especulativos que necesitan ser promovidos al estatus de leyes y que la ciencia no está influida por aspectos sociales, políticos o religiosos, son algunos de los aspectos de la naturaleza de la ciencia, que pueden interferir en la aceptación y comprensión de la TE (e.g. Rudolph y Stewart, 1998; Dagher y BouJaoude, 2005; Hokayem y BouJaoude, 2008; Lombrozo et al., 2008; Gregory, 2009; Kim y Nehm, 2010; Smith, 2010b).

En Chile, existen algunos antecedentes que ponen de manifiesto la poca cobertura que tuvo la enseñanza de la TE en la segunda mitad del siglo XX (Medel, 2008; Camus, 2009; Tamayo y González, 2010). Estos antecedentes muestran que la enseñanza de la TE estuvo ausente del currículo nacional de ciencias entre 1969 y 1985 (Medel, 2008; Camus, 2009; Tamayo y González, 2010). No obstante, hace ya más de dos décadas que este tema ha vuelto a los documentos oficiales de la educación en Chile. Esto, unido a la ausencia de un tratamiento explícito de temas como el Diseño Inteligente y la carencia de un movimiento creacionista organizado en Chile podría sugerir, según Medel (2008), un escenario positivo en cuanto a la aceptación y comprensión de la TE en la población. Además, la biología evolutiva es una disciplina vigorosa y productiva en Chile (para una revisión reciente véase Medel, 2008), lo cual se refleja en la gran diversidad de líneas de investigación que hoy existen, las cuales ya se han materializado incluso en libros sobre el tema (e.g. Medel et al., 2009; Gallardo, 2011; Veloso y Spotorno, 2012). No obstante, la poca cobertura y articulación que aun tiene el tema en la formación de profesores de biología (Camus, 2009) y de enseñanza básica (Vergara y Cofré, 2008), unido a la poca formación que tienen los profesores de ciencia en general en el tema de la naturaleza de la ciencia (Cofré et al., 2010, 2012), generan un escenario bastante más complejo y menos predecible sobre cuál es la comprensión y aceptación que tiene los estudiantes universitarios de la TE.

\section{PROPÓSITOS DE LA INVESTIGACIÓN}

De acuerdo a lo expuesto anteriormente, el principal objetivo de este estudio fue realizar una primera descripción y caracterización de cuál es la comprensión que tienen un grupo de estudiantes universitarios de carreras del área científico - biológica y pedagógica sobre la TE, que no han asistido a cursos de evolución a nivel universitario. La 
elección de este grupo de estudiantes se justifica ya que es un segmento de la población que se ha beneficiado directamente del nuevo currículo escolar que incorporó la TE en su forma más amplia a partir de 1999 (MINEDUC 1998, Camus 2009). De esta forma, las preguntas de investigación que guiaron este estudio fueron:

¿Cuál es el grado de comprensión que tienen estudiantes universitarios de primer año de carreras asociadas al área de las ciencias naturales de los conceptos de Ley y Teoría?

¿Cuál es el grado de aceptación de la Teoría de la Evolución que tienen estos estudiantes?

¿Cuál es el grado de comprensión de la Teoría de la Evolución que tienen estos estudiantes?

¿Cuál es el grado de correlación que existe entre estas tres variables?

\section{METODOLOGÍA}

\subsection{LOS SUJETOS}

La muestra estuvo constituida por 248 estudiantes que cursaban primer año de tres carreras del área científica o educativa, en tres universidades de Santiago, una de ellas pertenece al consejo de rectores (73 estudiantes) y las otras dos universidades de carácter privado (140 y 35 estudiantes cada carrera). En cada una de las carreras, el instrumento fue aplicado a la totalidad de alumnos pertenecientes a una corte inicial (primer o segundo año). En general, la gran mayoría de los estudiantes (casi un 60\%) provienen de colegios particulares subvencionados, siendo menor la representación de estudiantes provenientes de colegios municipales $(25 \%)$ o particulares pagados $(15 \%)$. De acuerdo a la malla curricular de las carreras participantes, ninguno de los estudiantes había tenido un curso específico sobre Evolución a nivel universitario. Por lo tanto, lo que se evaluó en términos de conocimientos, fue el grado de comprensión de la TE producto de la instrucción recibida en la enseñanza básica y media, por parte de los estudiantes, de acuerdo al currículo nacional de ciencias de 1998.

\subsection{INSTRUMENTO Y ANÁLISIS DE LOS DATOS}

La recolección de los datos se realizó aplicando un instrumento diseñado a partir de dos diferentes instrumentos ampliamente utilizados en la literatura para evaluar la comprensión y la aceptación de la teoría de la evolución por diferentes actores (Nehm y Schonfeld, 2007; Gregory y Ellis, 2009; Kim y Nehm, 2010). El instrumento utilizado para recoger los datos incluyó 18 preguntas: tres de carácter demográfico, 14 con una estructura de escala likert y una pregunta abierta. Las preguntas de escala likert abordaron 3 aspectos diferentes: la Comprensión sobre Teoría y Ley, CTL (tres preguntas), la Comprensión de la Teoría de la Evolución, CTE (ocho preguntas) y la Aceptación de la Teoría de la Evolución, ATE (tres preguntas) (Lombrozo et al., 2008; Gregory y Ellis, 2009; Kim y Nehm, 2010). La pregunta abierta se incluyó con el propósito de evaluar en una forma más profunda y detallada, cuál es la comprensión que poseen los estudiantes consultados sobre los mecanismos subyacentes a la TE (e.g. Kampourakis y Zogza, 2007; Nehm y Reilly, 2007; Gregory y Ellis, 2009). 
Aunque la confiabilidad del instrumento fue baja al analizar cada uno de los aspectos estudiados, (alpha de Cronbach CTL $=0.57$; alpha de Cronbach ATE $=0.59$; alpha de Cronbach CTE $=0,47$ ), estos resultados se han explicado en los instrumentos de origen por el reducido número de ítems utilizados, obteniéndose valores cercanos a 0.6 (e.g. Kim y Nehm, 2010).

Para el análisis de los datos cuantitativos, en primer lugar se realizó una inspección gráfica de cada una de las preguntas del cuestionario. Luego de este análisis, se procedió a agrupar las diferentes preguntas que constituyen cada variable, sumando sus valores. Finalmente, se exploró la correlación entre las tres variables estudiadas CTL, CTE y ATE. Todos los análisis se realizaron en el programa SPPS 18.0. Para el análisis cualitativo de la pregunta abierta se usaron categorías ya establecidas, las que caracterizaron los diferentes tipos de conocimientos que el estudiante podría demostrar al enunciar los mecanismos de la TE (Tabla 1). La generación de la rúbrica para cada tipo de conocimiento se obtuvo a través del análisis de la literatura sobre errores conceptuales de estudiantes en el tema de la TE y la Selección Natural (e.g. Nehm y Reilly, 2007; Kampourakis y Zogza, 2007; Gregory, 2009; Gregory y Ellis, 2009; Gutiérrez, 2009). Se consideró que la respuesta del estudiante era completa para una categoría cuando incluyó en su respuesta todos los aspectos descritos para ese tipo de conocimiento (Tabla 1). Si el estudiante mencionó alguno, pero no todos los aspectos de la rúbrica para un conocimiento, se consideró una respuesta incompleta. Aquellos estudiantes que incorporaron aspectos de más de una categoría fueron catalogados como una respuesta combinada. El análisis de las respuestas fue realizado por tres de los autores y se obtuvo un $93 \%$ de congruencia en el análisis. Las discrepancias se discutieron y se llegó a un consenso entre los tres investigadores en todas ellas.

Tabla 1. Mecanismos de evolución usualmente encontrados en las respuestas de estudiantes, sus marcos teóricos asociados, ejemplo de respuesta y la rúbrica completa con la cual se evaluó la pregunta abierta del cuestionario de conocimientos de evolución

\begin{tabular}{|c|c|c|c|}
\hline Marco Teórico & $\begin{array}{l}\text { Mecanismo } \\
\text { de origen del } \\
\text { cambio }\end{array}$ & Rúbrica completa & Ejemplo \\
\hline $\begin{array}{l}\text { Evidencias } \\
\text { fósiles y de } \\
\text { anatomía } \\
\text { comparada }\end{array}$ & $\begin{array}{l}\text { No se da un } \\
\text { mecanismo de } \\
\text { forma explicita, } \\
\text { sino que se } \\
\text { mencionan } \\
\text { evidencias } \\
\text { indirectas del } \\
\text { proceso de } \\
\text { evolución. }\end{array}$ & $\begin{array}{c}\text { El estudiante hace alusión a: } \\
\text { 1) la explicación se da por la } \\
\text { comparación de la especie } \\
\text { actual y el ancestro gracias } \\
\text { al registro fósil (anatomía) } \\
\text { o 2) la comparación de la } \\
\text { especie y el ancestro por } \\
\text { rasgos moleculares (DNA, } \\
\text { proteínas). }\end{array}$ & $\begin{array}{c}\text { Las aves han } \\
\text { evolucionado } \\
\text { de los reptiles } \\
\text { ya que se han } \\
\text { encontrado fósiles } \\
\text { de dinosaurios con } \\
\text { plumas (respuesta } \\
\text { amecanística } \\
\text { completa). }\end{array}$ \\
\hline
\end{tabular}




\begin{tabular}{|c|c|c|c|}
\hline $\begin{array}{l}\text { Selección } \\
\text { natural } \\
\text { (Darwin) }\end{array}$ & $\begin{array}{c}\text { Reproducción } \\
\text { y sobrevivencia } \\
\text { diferencia basada } \\
\text { en la variabilidad } \\
\text { poblacional. }\end{array}$ & $\begin{array}{l}\text { El estudiante hace alusión } \\
\text { a: 1) la existencia de } \\
\text { variación en la población } \\
\text { gracias a mutaciones al } \\
\text { azar, 2) reproducción y } \\
\text { sobrevivencia diferencial } \\
\text { de algunos individuos } \\
\text { de la población, 3) los } \\
\text { sobrevivientes traspasan } \\
\text { sus características a la } \\
\text { descendencia, y 4) en el } \\
\text { tiempo el \% de la población } \\
\text { que posee estos rasgos se } \\
\text { hace mayor (Modificado de } \\
\text { Jensen et al., 2007). }\end{array}$ & $\begin{array}{c}\text { Gracias a mutaciones } \\
\text { al azar cierta parte } \\
\text { de la población } \\
\text { del ancestro no } \\
\text { volador sobrevivió } \\
\text { y se reprodujo más } \\
\text { gracias a ciertos } \\
\text { rasgos asociados } \\
\text { al vuelo (respuesta } \\
\text { darwiniana } \\
\text { incompleta solo } \\
\text { aludiendo los } \\
\text { mecanismos de } \\
\text { generación del } \\
\text { cambio y no de } \\
\text { mantención). }\end{array}$ \\
\hline $\begin{array}{c}\text { Transformismo } \\
\text { (Lamarck) }\end{array}$ & $\begin{array}{l}\text { Causa debido al } \\
\text { uso o desuso de } \\
\text { las estructuras. }\end{array}$ & $\begin{array}{l}\text { El estudiantes hace } \\
\text { alusión a: 1) la mayor } \\
\text { frecuencia en el uso de } \\
\text { los órganos o el desuso } \\
\text { de ellos lleva a un mayor } \\
\text { desarrollo o pérdida de los } \\
\text { mismos y 2) esta pérdida } \\
\text { o desarrollo de órganos se } \\
\text { traspasa a la generación } \\
\text { venidera (Modificado de } \\
\text { Kampourakis y Zogza, } \\
\text { 2007). }\end{array}$ & $\begin{array}{l}\text { Como el ancestro de } \\
\text { los topos no usaba } \\
\text { sus ojos, finalmente } \\
\text { la especie los fue } \\
\text { perdiendo (respuesta } \\
\text { lamarckiana } \\
\text { incompleta sin aludir } \\
\text { explícitamente } \\
\text { a la transmisión } \\
\text { de caracteres } \\
\text { adquiridos). }\end{array}$ \\
\hline $\begin{array}{c}\text { Intuitivo } \\
\text { (Teleológico } \\
\text { - Ortogénesis) }\end{array}$ & $\begin{array}{c}\text { Causa final dada } \\
\text { por una voluntad } \\
\text { del organismo } \\
\text { para responder a } \\
\text { los cambios del } \\
\text { medio. }\end{array}$ & $\begin{array}{l}\text { El estudiante hace alusión } \\
\text { a: 1) la existencia de una } \\
\text { voluntad del organismo por } \\
\text { responder a una necesidad } \\
\text { del entorno (Modificado } \\
\text { de Kampourakis y Zogza, } \\
\text { 2007). }\end{array}$ & $\begin{array}{l}\text { Las bacterias de } \\
\text { hoy en día son } \\
\text { difíciles de matar ya } \\
\text { que ellas cambian } \\
\text { en respuesta a los } \\
\text { nuevos antibióticos } \\
\text { (respuesta intuitiva } \\
\text { completa). }\end{array}$ \\
\hline
\end{tabular}

\section{RESULTADOS}

\subsection{EL CONOCIMIENTO SOBRE LOS CONCEPTOS DE TEORÍA Y LEY}

En cuanto a la comprensión que tienen los estudiantes de los conceptos de Teoría y Ley, los resultados muestran una confusión a la hora de distinguir entre ellos y 
comprender sus verdaderos alcances. La Figura 1 muestra la distribución de respuesta de los estudiantes a las tres proposiciones de la variable CTL. Por ejemplo, la proposición que dice: "En ciencia, el conocimiento que comienza como una Teoría puede llegar a convertirse en una Ley una vez que se acumula una gran cantidad de evidencia que lo apoya", muestra una gran proporción de respuesta de acuerdo o muy de acuerdo (72\%), una menor cantidad inseguros $(21 \%)$ y muy pocos estudiantes en desacuerdo (7\%). Sin embargo, cuando se les propone otra oración con una explicación más correcta de cuál es la relación entre Ley y Teoría: "En ciencia, las teorías no se convierten en leyes, sino que son explicaciones de los fenómenos naturales", las respuestas de los estudiantes tienden a distribuirse de una forma más equivalente a través de los diferentes grados de acuerdo, lo que se contradice con lo esperado según la primera respuesta. Finalmente, al analizar la proposición: "La evolución es 'sólo una teoría', así como la teoría del creacionismo", la cual pone en el contexto de la TE, la aplicación del conocimiento sobre naturaleza de la ciencia, se observa que existe un cuarto de los estudiantes que está de acuerdo, por lo que se infiere que no comprenden el significado de Teoría Científica que posee el conocimiento evolutivo, a diferencia del Creacionismo (Figura 1).

\subsection{LA ACEPTACIÓN DE LA TEORÍA DE LA EVOLUCIÓN}

En cuanto a la aceptación de la TE como explicación científica, la gran mayoría de los estudiantes mostró un alto grado de acuerdo. La Figura 2 muestra el grado de acuerdo a las tres proposiciones que se incluyeron en la variable ATE. Por ejemplo, a la proposición: "El parentesco de las especies, por medio de la descendencia de un ancestro común, a través de la evolución, es un hecho científico establecido...", se puede ver que cerca del $74 \%$ del total de estudiantes está de acuerdo o muy de acuerdo con esta proposición, casi un $19 \%$ no está seguro y sólo cerca de un 7\% está en desacuerdo. Un resultado diferente, pero en congruencia con lo anterior, se encontró en las respuestas a la proposición que la evolución sólo es una especulación (Figura 2). Aquí el 79\% estuvo en desacuerdo o muy en desacuerdo, un $12 \%$ no estaba seguro y un $9 \%$ estaba de acuerdo o muy de acuerdo. Finalmente, la distribución de frecuencia de respuestas a la proposición: La única prueba de la evolución es el registro fósil, y como el registro fósil es incompleto la teoría de evolución no es correcta, es muy congruente con la pregunta anterior (11\% está de acuerdo o muy de acuerdo, $21 \%$ no está seguro y un $68 \%$ está en desacuerdo o muy en desacuerdo) (Figura 2).

\subsection{LA COMPRENSIÓN DE LA TEORÍA DE LA EVOLUCIÓN}

En cuanto a la comprensión de la TE, se observó que un porcentaje importante de estudiantes presenta algunos de los errores conceptuales más frecuentemente descritos en la literatura. La Figura 3 muestra la distribución del grado de acuerdo de los estudiantes a las ocho preguntas que formaron parte de la variable CTE. Esta variable incluyó cuatro errores conceptuales muy frecuentes. Las concepciones de los estudiantes en cuanto a estos cuatro errores mostró distintos grados acuerdo. Por ejemplo, a la proposición: " $\mathrm{La}$ evolución siempre va de lo más simple a lo más complejo", un 50\% de los estudiantes estuvo de acuerdo o muy de acuerdo con ella, mientras que casi un $35 \%$ de ellos no tuvo claro la veracidad de esta proposición. Un resultado similar ocurre cuando se les 
Figura 1. Gráfico que muestra la distribución de respuestas de alumnos a las tres preguntas incluidas en la variable CTL: Comprensión sobre Teoría y Ley. El color negro muestra el \% de alumnos que responde 5 ó 4 , el color blanco muestra el \% de estudiantes que responde 3 y el color gris el $\%$ de estudiantes que responde 1 ó 2 , siendo 5 muy de acuerdo y 1 muy en desacuerdo

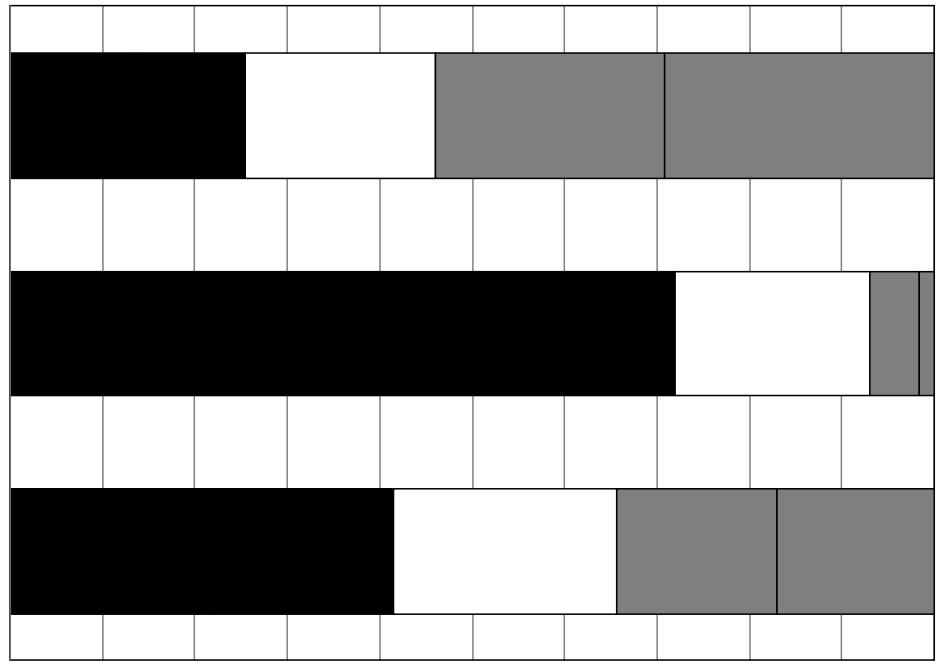

Figura 2. Gráfico que muestra la distribución de respuestas de alumnos a las tres preguntas incluidas en la variable ATE: Aceptación de la Teoría de la Evolución. El color negro muestra el $\%$ de alumnos que responde 5 ó 4 , el color blanco muestra el \% de estudiantes que responde 3 y el color gris el $\%$ de estudiantes que responde 1 ó 2 , siendo 5 muy de acuerdo y 1 muy en desacuerdo

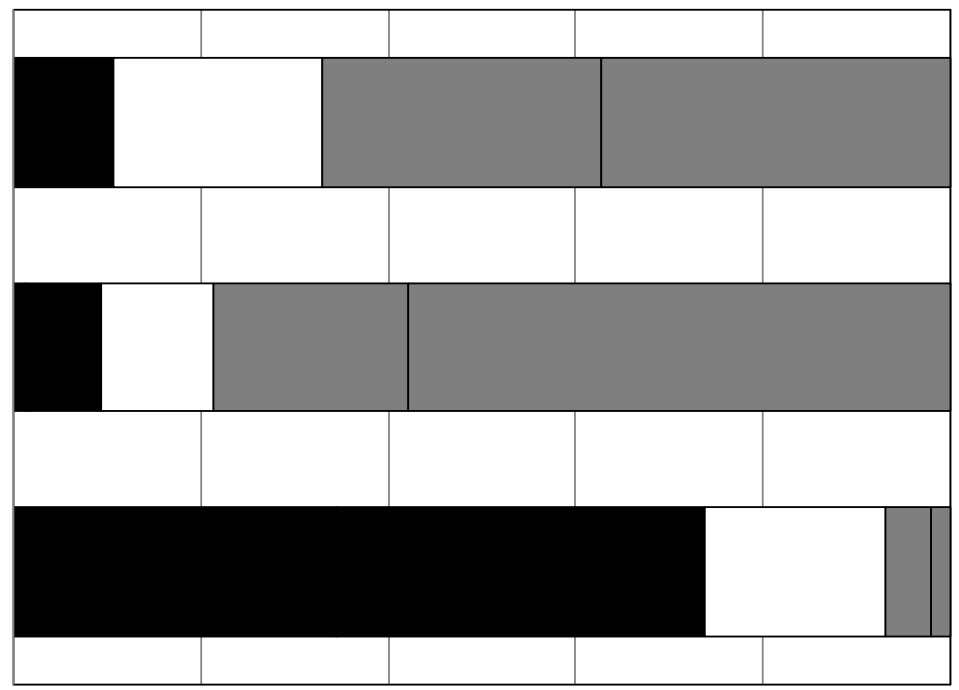


propuso: "El hombre no desciende directamente de los primates actuales como el chimpancé o el gorila", donde casi un $60 \%$ de los encuestados no está de acuerdo o no está seguro (Figura 3). En cuanto a las otras dos proposiciones, el grado de acuerdo con los errores conceptuales fue muy bajo, demostrando un mayor conocimiento de dichos temas. Específicamente, se llegó a un $93 \%$ de desacuerdo con: la evolución es un proceso que ocurrió solo en el pasado y a un $91 \%$ de acuerdo con: El proceso de evolución afecta a los humanos como cualquier otro organismo (Figura 3).

En cuanto a los mecanismos que explican el cambio de las especies y los procesos de adaptación que resultan, una proporción importante de estudiantes dice estar de acuerdo con los mecanismos Darwinistas que explican el proceso (casi un 60\% en la pregunta sobre evolución de la rapidez en el Cheetha y más de un $70 \%$ en la pregunta sobre evolución de la resistencia en bacterias). Sin embargo, cuando se les presenta una explicación alternativa de tipo Teleológica - intuitiva, donde el principal mecanismo es la necesidad creada por el ambiente y la voluntad del organismo, también existe una gran proporción de estudiantes que dicen estar de acuerdo con ella (Figura 3).

Como resumen de esta evaluación cuantitativa de la comprensión de los estudiantes o de la presencia de errores conceptuales, se puede decir que, en general, cuando uno agrupa todos los valores de las ocho respuestas, no más de un $22 \%$ de los estudiantes responde de forma correcta a las ocho preguntas sobre conocimiento de evolución. Una gran cantidad de estudiantes $(57 \%)$ responde a las preguntas de forma inconsistente o no está seguro de sus respuestas, mientras cerca de un $21 \%$ estuvo de acuerdo con la mayoría de los errores conceptuales o en desacuerdo con proposiciones correctas.

Esta falta de comprensión de los mecanismos Darwinistas que explican la Evolución por parte de los estudiantes, se ve aun más clara cuando se analizan los resultados de las respuestas abiertas a la pregunta: “...explique cómo cree usted que los biólogos evolutivos han explicado la evolución del murciélago a partir de un ancestro parecido a un roedor". En la Tabla 2 se muestra el número de respuestas y el porcentaje de los diferentes tipos de respuestas reconocidas. Aquí se puede ver que sólo un 7\% de las 200 respuestas esbozan, al menos alguna parte del mecanismo Darwinistas de evolución acerca de la reproducción y sobrevivencia diferencial basada en la variabilidad poblacional. Por otra parte, se pudo constatar la ausencia total de respuestas que tienen que ver con la explicación Lamarckistas de la evolución. De esta forma, las respuestas más frecuentes tienen que ver con explicaciones de tipo intuitivas (necesidad y/o voluntad de la especie o el organismo) o amecanísticas. En estas últimas, los estudiantes no explicaron cómo habría sido la evolución del murciélago, sino que mencionaron evidencias aisladas que apoyarían este proceso, como la presencia de fósiles con características intermedias o semejanzas anatómicas entre las distintas especies comparadas. 
Figura 3. Gráfico que muestra la distribución de respuestas de alumnos a las ocho preguntas incluidas en la variable CTE: Aceptación de la Teoría de la Evolución. El color negro muestra el $\%$ de alumnos que responde 5 ó 4, el color blanco muestra el \% de estudiantes que responde 3 y el color gris el \% de estudiantes que responde 1 ó 2 , siendo 5 muy de acuerdo y 1 muy en desacuerdo.

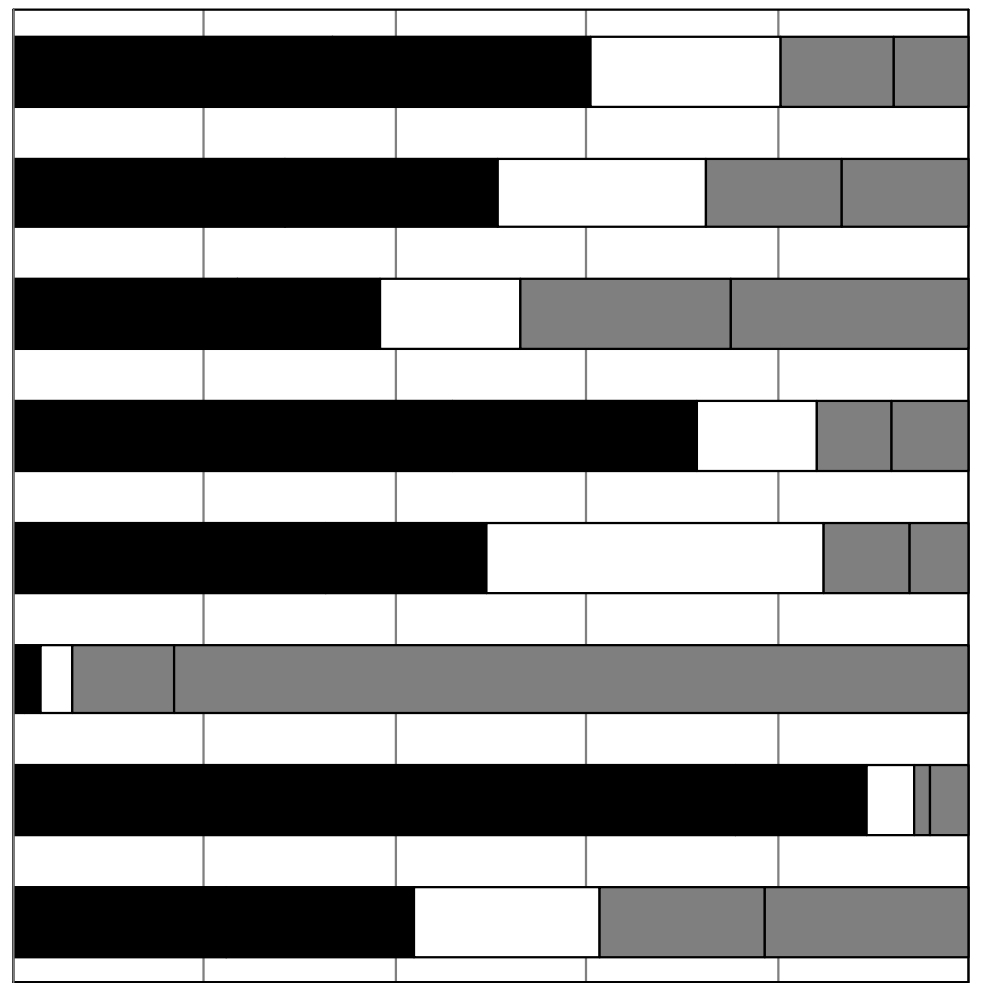

Tabla 2. Frecuencia y porcentaje de cada una de las categorías de respuestas que explican la evolución reconocidas en este trabajo

\begin{tabular}{|c|c|c|c|c|}
\hline \multirow{2}{*}{$\begin{array}{c}\text { Tipo de } \\
\text { respuestas }\end{array}$} & \multicolumn{2}{|c|}{$\begin{array}{c}\text { Número de respuestas por } \\
\text { tipo }\end{array}$} & $\begin{array}{c}\text { Total de } \\
\text { respuestas }\end{array}$ & Porcentajes (\%) \\
\cline { 2 - 3 } & Completa & Incompleta & & \\
\hline Darwinista & 2 & 12 & 14 & 7 \\
\hline Lamarckista & 0 & 0 & 0 & 0 \\
\hline $\begin{array}{c}\text { Intuitiva - } \\
\text { necesidad }\end{array}$ & 66 & 8 & 74 & 37 \\
\hline Amecanística & 37 & 21 & 58 & 29 \\
\hline Combinaciones & & & 17 & 8,5 \\
\hline Otra & & & 3 & 1,5 \\
\hline No sabe & & & 34 & 17 \\
\hline
\end{tabular}




\section{RELACIONES ENTRE VARIABLES}

Como una primera exploración sobre las posibles causas o relaciones complejas entre las diferentes variables estudiadas en este trabajo, se realizó un análisis de correlación entre las variables que daban cuenta de la aceptación y comprensión de la TE y la comprensión de los conceptos de Teoría y Ley que mostraron los estudiantes. Este análisis mostró que no existe una correlación aparente entre la CTE, ATE y CTE (Tabla 3).

Tabla 3. Coeficientes de Correlación de Pearson entre las variables estudiadas. No se observo correlación entre las variables estudiadas

\begin{tabular}{|l|l|l|l|}
\hline Variables CTL ATE CTE \\
\hline Comprensión sobre Teoría y Ley (CTL) & 1 & -0.138 & 0.055 \\
\hline Aceptación de la Teoría de la Evolución (ATE) & - & 1 & 0.071 \\
\hline Comprensión de la Teoría de la Evolución (CTE) & - & - & 1 \\
\hline
\end{tabular}

\section{DISCUSION Y CONCLUSIONES}

6.1. ¿CUÁL ES EL GRADO DE COMPRENSIÓN QUE TIENEN ESTUDIANTES UNIVERSITARIOS DE PRIMER AÑO DE CARRERAS ASOCIADAS AL ÁREA DE LAS CIENCIAS NATURALES DE LOS CONCEPTOS DE LEY Y TEORÍA?

En este trabajo se ha mostrado que gran parte del grupo de estudiantes analizados no posee una comprensión clara del significado de lo que es una teoría científica. Esta falta de comprensión se aprecia no solo en términos del manejo de los conceptos de Teoría y Ley, sino también en su aplicación a la hora de reconocer qué tipo de conocimiento es una teoría científica y cuál no. Estos resultados coinciden ampliamente con lo descrito en la literatura, donde se ha demostrado que la comprensión de los conceptos de Teoría y Ley es uno de los principales problemas de la enseñanza de la naturaleza de la ciencia (Lederman, 2007). Por ejemplo, en un estudio muy similar al realizado en este trabajo, Gregory y Ellis (2009) mostró una incongruencia similar entre las respuestas de estudiantes de postgrado del ámbito biológico. Si bien más de un $70 \%$ estaba de acuerdo con que las teorías no se convierten en leyes, de todas formas cerca de un $60 \%$ estaba de acuerdo con que las teorías podrían hacerlo al acumular más evidencia empírica. Esto daba como resultado que, al menos un cuarto de los 187 estudiantes poseían una mala comprensión de lo que es una teoría científica.

\section{2. ¿CUÁL ES EL GRADO DE ACEPTACIÓN DE LA TEORÍA DE LA EVOLUCIÓN QUE TIENEN LOS ESTUDIANTES?}

En este estudio se encontró que la mayoría de los estudiantes está de acuerdo con que la TE es un conocimiento científico establecido. Esto coincide con la literatura internacional donde se ha mostrado que el grupo de la población que posee el mayor grado de aceptación de la TE es el de los estudiantes universitarios (e.g. Mc Fadden et al., 2007; Gregory y Ellis, 2009; Gregory, 2009). Por ejemplo, Gregory y Ellis (2009) 
encontraron que un $70 \%$ de los estudiantes de postgrado investigados aceptaba la TE. Es importante señalar que, a diferencia de nuestro estudio, cerca de la mitad de los estudiantes de la investigación de Gregory y Ellis (2009) ya habían tenido algún curso de evolución, a diferencia de nuestro grupo. Si bien estos resultados no son extrapolables al resto de la población, esta primera evaluación podría servir para levantar una hipótesis de trabajo sobre cuál sería la aceptación de la TE que tiene el público general en Chile. De este trabajo se desprende que la aceptación debería ser menor al $74 \%$, ya que existen antecedentes que personas de mayor edad tienden a aceptar menos que los jóvenes la TE (Mc Fadden et al., 2007). Por lo tanto, es probable que en Chile exista una aceptación superior al $60 \%$, que es lo que se encuentra en la mayoría de los países de Europa (Miller et al., 2006). De esta forma, nuestro trabajo permite hacer una predicción bastante más informada y certera de la que se ha generado antes en base a antecedentes más bien indirectos (e.g. Medel, 2008).

\section{3. ¿CUÁL ES EL GRADO DE COMPRENSIÓN DE LA TEORÍA DE LA EVOLUCIÓN QUE TIENEN} LOS ESTUDIANTES?

En este trabajo hemos evidenciado que los estudiantes presentan una gran falta de comprensión de los mecanismos que explican la evolución. La mayoría de los estudiantes estuvieron de acuerdo con 4 de los 8 errores conceptuales que se presentaron en el cuestionario, mientras que cuando se les pidió dar una explicación coherente sobre un proceso evolutivo particular, las explicaciones a través de mecanismos Darwinistas no sobrepasaron el $10 \%$. Este último resultado es muy similar a lo descrito por Kampourakis y Zogza (2007) para estudiantes de enseñanza secundaria en Grecia. Estos autores mostraron que del total de estudiantes a los que se les pidió explicar la evolución de la jirafa, menos del 5\% fueron capaces de incluir algún aspecto de los mecanismos incluidos en la explicación Darwinista. Resultados algo diferentes encontraron Gregory y Ellis (2009) para estudiantes universitarios en Canadá. Estos autores encontraron que más de un $70 \%$ de los estudiantes evidenciaban la comprensión de mecanismos Darwinistas de evolución de acuerdo a preguntas cerradas. No obstante, las respuestas abiertas a preguntas sobre mecanismos evolutivos, mostraron una mayor dispersión, desde una gran capacidad explicativa (70\% de las respuestas a la pregunta de evolución de peces sin ojos) a una baja capacidad de articular mecanismos Darwinistas (33\% de las respuestas a la pregunta de la evolución de los murciélagos). Un resultado similar a este último se ha mostrado también en visitantes de museos de USA, donde no más del $30 \%$ de los encuestados dio una explicación Darwinista a la evolución de la rapidez del Cheetah (Mc Fadden et al., 2007).

En cuanto a los otros tipos de explicaciones que dan los estudiantes, en nuestro trabajo hemos mostrado que la mayor representación corresponde a respuestas de tipo intuitivas que incluyen aspectos teleológicos (37\%) y que existe una ausencia total de explicaciones genuinamente Lamarkistas sobre uso y desuso de órganos. Este escenario es muy similar a lo encontrado por Kampourakis y Zogza (2007), donde se describen un $53 \%$ de respuestas intuitivas y un $16 \%$ a respuestas amecanísticas. Otros estudios (e.g. Mc Fadden et al., 2007; Gregory y Ellis, 2009; Pazza et al., 2010) también han mostrado que la mayoría de las respuestas no Darwinistas son de tipo intuitivas o amecanísticas, con una muy baja representación de explicaciones genuinamente Lamarckistas. Estos resultados también son corroborados por Gutiérrez (2009), quien en su interesante monografía muestra que, tanto en Norteamérica como en España, una gran cantidad de 
estudiantes universitarios y secundarios consideran los cambios evolutivos como resultado de la necesidad impuesta por el ambiente.

Estos resultados sobre la comprensión de la TE por parte de estudiantes universitarios en Chile, develan un escenario sobre la enseñanza y el aprendizaje de la evolución en la educación secundaria bastante más negativo que proposiciones anteriores (e.g. Camus, 2009; Medel, 2008).

\section{4. ¿EXISTE ALGÚN GRADO DE CORRELACIÓN ENTRE ESTAS TRES VARIABLES?}

Existen evidencias en la literatura, que relacionan la comprensión de la naturaleza de la ciencia y el grado de aceptación que demuestran los estudiantes hacia la TE (Dagher y BouJaoude, 2005; Hokayem y BouJaoude, 2008; Lombrozo et al., 2008; Gregory, 2009; Kim y Nehm, 2010; Smith, 2010b). Esto se expresa en que muchas veces los estudiantes tienden a pensar que la TE es un conocimiento que aun debe ser probado, el cual carece de evidencia empírica y que incluso puede tener el mismo estatus que las proposiciones no científicas como el Creacionismo (e.g. Dagher y BouJaoude, 2005; Hokayem y BouJaoude, 2008; Gregory, 2009; véase también Smith, 2010b para una revisión). Por ejemplo, la frase "la evolución es solo una teoría" (evolution is just a theory) es una de las más usadas por los sujetos que poseen este error conceptual (e.g. National Academy of Sciences, 1998; Camus, 2009; Gregory, 2009; Smith, 2010b). Si bien en nuestro análisis hubo evidencias tanto de que los estudiantes no poseen una buena comprensión de lo que es una teoría científica, como la presencia de muchos errores conceptuales sobre el tema, el análisis correlacional no evidenció una relación significativa entre ninguna de las variables estudiadas. Esta falta de relación coincide con algunos estudios cuantitativos hechos en Norteamérica (e.g. Nehm et al., 2009; Smith, 2010b). Sin embargo, otros estudios, incluyendo algunos de tipo cualitativos, han mostrado que estudiantes que no creen o no comprenden la TE, muchas veces también poseen una visión restringida y poco adecuada de lo que es el quehacer científico (e.g. Dagher y BouJaoude, 2005; Hokayem y BouJaoude, 2008; Gregory, 2009; Kim y Nehm, 2010). Por lo tanto, aun se debe seguir explorando las relaciones complejas que existen entre estas variables (Smith, 2010b).

\subsection{RECOMENDACIONES}

A pesar de las limitaciones que tiene nuestro trabajo, muchas de ellas propias de un estudio exploratorio y descriptivo sobre un tema desconocido en Chile, es posible realizar algunas recomendaciones y sugerencias que pueden guiar futuras investigaciones en el tema.

Por una parte, es claro que un segundo paso es explorar la comprensión de la TE en otros grupos, por ejemplo, el público en general, los estudiantes de enseñanza media o en profesores básicos y de enseñanza media (Biología). Este último grupo es el principal responsable de la enseñanza y aprendizaje de la TE (Camus, 2009), por lo tanto es imprescindible volcar los esfuerzos a evaluar los conocimientos y concepciones de profesores de Biología en ejercicio, así como también a mejorar su preparación en el tema, tanto a nivel de pregrado como en la formación continua.

Un segundo punto que emerge como una necesidad prioritaria es aplicar y desarrolla más y mejores instrumentos para describir y evaluar la comprensión que tiene la población en Chile de la TE. Si bien, el instrumento utilizado en este estudio es muy 
similar a aquellos ampliamente utilizados en investigaciones internacionales (e.g. Mc Fadden et al., 2007; Kampourakis y Zogza, 2007; Gregory y Ellis, 2009), existen otros instrumentos que podrían mejorar nuestra capacidad de caracterizar la aceptación (e.g. MATE, [Rutledge y Sadler, 2007]) y comprensión de la TE (ECK [Nehm y Schonfeld, 2007]), así como la comprensión de la naturaleza de la ciencia (VNOS [Lederman et al. 2002]) y su aplicación al conocimiento de evolución (ENOS[Nehm y Schonfeld, 2007]). Además, el uso de entrevistas (e.g. Dagher y BouJaoude, 2005; Hokayem y BouJaoude, 2008) podría develar detalles sobre el origen y las características de los errores conceptuales que poseen los estudiantes, y de cómo las diferentes variables estudiadas en este trabajo pueden estar o no relacionadas.

En tercer lugar, y quizá lo más importante, teniendo claro tras esta investigación que la comprensión de los postulados de Darwin es el principal problema que tienen nuestros estudiantes para llegar a una real alfabetización en el tema, es indispensable comenzar a realizar estudios sobre cómo mejorar nuestra forma de enseñar la TE. Un primer aporte que surge de esta investigación, es el hecho de haber caracterizado algunos de los errores conceptuales que tienen los estudiantes sobre los mecanismos y procesos evolutivos. Si bien las misconpcetions que presentan estudiantes de diferentes niveles sobre la TE han sido extensamente caracterizadas (e.g. Alter y Nelson, 2002; Gregory, 2009; Gutiérrez, 2009), también se ha descrito que ellas rara vez son tomadas en cuenta para desarrollar una enseñanza eficiente de la TE (e.g. Alter y Nelson, 2002; Gregory, 2009; Gutiérrez, 2009). Para profundizar en este tema será imprescindible realizar estudios más complejos donde, por una parte, se lleven a cabo diseños cuasi-experimentales para evaluar la efectividad de diferentes estrategias de enseñanza y, por otra parte, también se realicen estudios cualitativos a fondo que describan en profundidad la complejidad de las preconcepciones que mantienen los estudiantes, así como cuales son las prácticas que hoy en día realizan los profesores en el ámbito de la enseñanza de la TE.

\section{REFERENCIAS BIBLIOGRÁFICAS}

Alter, B., y Nelson, G. E. (2002). Perspective: teaching evolution in higher education. Evolution, vol. 56, n. 10, 1891-1901.

Camus, P. (2009). Educación Científica y Evolutiva en Chile. Problemas funcionales y conflictos entre enseñar y aprender. Gayana, n. 73, (Suplemento), 19-31.

Cofré, H., J. Camacho, A., Galaz, J. Jiménez, D. Santibáñez y Vergara, C. (2010). La educación Científica en Chile: debilidades de la enseñanza y futuros desafíos de la educación de profesores de ciencia. Estudios Pedagógicos, n. 26, 279-293.

Cofré, H., C. Vergara, J. Jiménez, D. Santibáñez, J. Camacho y Galaz, A. (2012). Future challenges of science teacher education in Chile. Science Teacher Education, n. 63, 8-18.

Dagher, Z. R., y BouJaoude, S. (2005). Students' perceptions of the nature of evolutionary theory. Science Education, n. 89, 378-391.

Dobzhansky, T. (1973). Nothing in Biology makes sense except in the light of evolution. The American Biology Teacher, vol. 35, n. 3, 125-129.

Gallardo, M. H. (2011). Evolución: El curso de la vida. Buenos Aires: Editorial Médica Panamericana. Gregory, T. R. (2009). Understanding Natural Selection: Essential Concepts and Common Misconceptions. Evolution: Education and Outreach, n. 2, 156-175.

Gregory, T. y Ellis, C. (2009). Conceptions of evolution among science graduate students. Bioscience, n. 59, 792-799. 
Gutiérrez, A. (2009). Biología. La teoría de la evolución en la escuela. Buenos Aires: Editorial Biblos. Hokayem, H., y BouJaoude, S. (2008). College students' perceptions of the theory of evolution. Journal of Research in Science Teaching, n. 45, 395-419.

Harlen, W. (2010). Principles and big ideas of science education. ASE. Web: www.ase.org.uk.

Jensen, M., Moore, R., Hatch, J., y Hsu, L. (2007). Scoring rubric for students' responses to simple evolution questions: Darwinian components. The American Biology Teacher, n. 69, 394-396 $+398-399$.

Kampourakis K, y Zogza V. (2007). Students' preconceptions about evolution: How accurate is the characterization as "Lamarckian" when considering the history of evolutionary thought? Science and Education, n. 16, 393-422 .

Kim, S. Y. y Nehm, R. H. (2010). A cross-cultural comparison of Korean and American science teachers' views of evolution and the nature of science. International Journal of Science Education, n. 10, 1-31.

Lederman, N. G., Abd-El-Khalick, F., Bell, R. L., y Schwartz, R. (2002). Views of nature of science questionnaire (VNOS): Toward valid and meaningful assessment of learners' conceptions of nature of science. Journal of Research in Science Teaching, n. 39, 497-521.

Lederman, N.G. (2007). Nature of science: Past, present, and future. En S.K. Abell y N.G. Lederman (Eds.), Handbook of research on science education (pp. 831-879). Mahwah, NJ: Lawrence Erlbaum.

Lombrozo, T., A. Thanukos y Weisberg, M. (2008). The importance of understanding the nature of science for accepting evolution. Evolution: Education and Outreach, n. 1, 290-298.

López, P., y Spotorno A. (2008). $8^{\circ}$ Básico, Evolución. Material elaborado en el marco del programa Educación en Ciencias Basada en la Indagación (ECBI), por el Ministerio de Educación y la Universidad de Chile. Santiago de Chile: Ministerio de Educación.

Mayr, E. (2001). What evolution is. London: Weidenfeld \& Nicolson.

McFadden, B. B. A. Dunckel, S. Ellis, L. D. Dierking, L. Abraham-Silver, J. Kisiel y Koke, J. (2007). Natural history museum visitors' understanding of evolution. Bioscience, n. 57, 875-882.

Medel, R. (2008). The evolution of evolutionary thinking in Chile. Evolution: Education \& Outreach, n. 1, 318-322.

Medel, R., M. A. Aizen y Zamora, R. (Eds.) (2009). Ecología y evolución de interacciones plantaanimal. Santiago de Chile: Editorial Universitaria.

Miller, J.D., Scott, E. C., y Okamoto S. (2006). Public acceptance of evolution. Science, n. 313: $755-756$.

MINEDUC, (1998). Marco curricular de la educación media. Objetivos fundamentales y contenidos mínimos obligatorios de la educación media. Santiago de Chile: Ministerio de Educación.

MINEDUC, (2009). Ajuste curricular en Chile. Unidad de currículum y evaluación. Santiago de Chile: Ministerio de Educación.

More, A. (2008). Science teaching must evolve. Nature, n. 453, 31-32.

National Academy of Sciences, Working Group on Teaching Evolution. (1998). Teaching about evolution and the nature of science. Washington, DC: National Academy Press.

Nehm, R. H., y Schonfeld, I. S. (2007). Does increasing biology teacher knowledge of evolution and the nature of science lead to greater preference for the teaching of evolution in schools? Journal of Science Teacher Education, n. 18, 699-723.

Nehm, R.H. y Reilly, L. (2007). Biology majors' knowledge and misconceptions of natural selection. BioScience, n. 57, 263-272.

Nehm, R. H., Kim, S., y Sheppard, K. (2009). Academic preparation in biology and advocacy for teaching evolution: Biology versus non-biology teachers. Science Education, n. 93, 1122-1146.

Pazza, R., P. R. Penteado, y Kavalco, K. F. (2010). Misconceptions about evolution in Brazilian freshmen students. Evolution: Education \& Outreach, n. 3, 107-113. 
Rudolph, J.L y Stewart, J. (1998). Evolution and the nature of science: on the historical discord and its implications for education. Journal of Research in Science Teaching, $n$. 35, 1069-1089.

Rutledge, M. L., y Sadler, K. C. (2007). Reliability of the measure of acceptance of the theory of evolution (MATE) instrument with university students. The American Biology Teacher, $n$. $51,275-280$.

Smith, M. U. (2010a). Current status of research in teaching and learning evolution: II. Pedagogical Issues. Science \& Education Special Darwinian Anniversary Year Issue, n. 19, 539-557.

Smith, M. U. (2010b). Current status of research in teaching and learning evolution: I. Philosophical/ Epistemological Issues Science \& Education Special Darwinian Anniversary Year Issue, $n$. 19, 523-538.

Spotorno, A. (1991). Origen y evolución de la especie humana. Santiago de Chile: Serie Científica Básica No 4, Centro de Extensión Biomédica, Fac. de Medicina.

Tamayo M., y González, F. (2010). La enseñanza de la evolución en Chile. Historia de un conflicto documentado en los textos de estudio de enseñanza media. Investigações e $m$ Ensino de Ciencias, $n$. 15, 310-336.

Veloso, A. y Spotorno, A. (Eds.) (2012). Darwin y la evolución: avances en la Universidad de Chile. Santiago de Chile: Editorial Universitaria.

Vergara, C. y Cofré, H. (2008). La enseñanza de las Ciencias Naturales en la Educación Básica chilena: un camino por recorrer. Revista Foro Educacional, n. 14, 85-104. 
